\title{
INTRODUCING TRAINING RELATED TO THE USE OF DRUGS TO PROTECT HUMANS FROM HIV INFECTION
}

\author{
A. Peña-Fernández ${ }^{1}, M^{\mathrm{a}}$ Ángeles Peña ${ }^{2}$, M.D. Evans ${ }^{1}$ \\ ${ }^{1}$ School of Allied Health Sciences, De Montfort University (UNITED KINGDOM) \\ ${ }^{2}$ Universidad de Alcalá, Departamento de Ciencias Biomédicas (SPAIN)
}

\begin{abstract}
Human immunodeficiency virus (HIV) chemoprophylaxis includes pre- and post-exposure prophylaxis (PrEP/PEP). PrEP has been shown to be effective in preventing HIV for high risk groups such as men who have sex with men, discordant heterosexual couples and people who inject drugs. However, appropriate education is required to successfully use these prevention tools. Thus, appropriate adherence in the use of PrEP is critical for success. Moreover, PrEP users should avoid risky behaviours that appear safe because of the PrEP regime. To increase awareness about HIV chemoprophylaxis, we have created a brief educational short course ( 3 hours) to discuss and study this important health topic with second year Medical Science (BMedSci) (Hons) students at De Montfort University (DMU, Leicester, UK) in collaboration with the University of Alcalá (Spain). The BMedSci at DMU is a three-year, full time degree, which provides a knowledge of human anatomy and physiology, enables students to undertake research in form of a final year research project and facilitates their understanding of the causes and mechanisms of human disease. This degree prepares students for a wide range of possible careers including applying for medicine, undertaking further postgraduate study to become qualified health professionals or PhD study, or work in hospitals as project managers. DMU students enrolled in the level 5 module "Evidence Based Medicine" $(n=41)$ completed a research-led workshop (2 hours) to identify interventions to reduce HIV infection rates in the Leicester community (including DMU students) following evidence-based public health methodology. To overcome time limitations, students were provided with the latest articles on HIV. Students designed their interventions in small groups and discussed them with the rest of the class. Prior to this short educational course, the students received a one hour lecture with up-to-date information about the HIV virus, infection routes and prevention (including HIV chemoprophylaxis), focussing on England and the UK Midlands (the UK region in which DMU is based). The current status about PrEP and the UK National Health Service (NHS) was also shown to encourage students' reflection and discussion. A feedback questionnaire was distributed to evaluate this first attempt to learn HIV prevention and protection, and improve awareness about this important infectious disease. BMedSci students showed real interest in this topic, as indicated by the extensive discussions generated during the workshop. $85 \%$ of participants indicated understanding the necessity of a global intervention to fight HIV and $92 \%$ of students reported to have learnt how to establish public health interventions to reduce HIV transmission. Some degree of confusion was evident with the students regarding transmission routes for HIV and public resources available in the community for earlier diagnosis of infection. BMedSci students also showed a lack of knowledge of preventative measures (PrEP and PEP) and who should be HIV screened and when. We believe that further courses and/or training to increase HIV awareness are critical for these students as they are destined to work in the health sector. To overcome time limitations in this degree, we are developing a complete on-line unit about HIV which will be available through the DMU website later in 2018. The approach and materials described in this paper may be relevant to provide training to other students at DMU and other European universities to prevent HIV infections and improve and promote healthy sex behaviours.
\end{abstract}

Keywords: HIV, chemical prophylaxis, PrEP, preventive education.

\section{INTRODUCTION}

HIV infection continues to be a public health problem despite preventive interactions, recent statistics from Public Health England [1] show that new diagnoses of HIV peaked in 2005 in the UK, diminishing over subsequent years; $5 \%$ of new diagnoses with HIV nationally in 2014 were in the East Midlands, the region in which Leicester is located. A steady increase in the number of people seen for HIV care has been noted over the 10 year period 2005 to 2015, a trend mirrored in the East Midlands with those affected receiving anti-retroviral therapy. 
Condom use remains the predominant prophylactic intervention to control rates of HIV infection. However, the use of drugs as prophylactic agents (chemoprophylaxis) for HIV infection is widely available since the development of effective anti-retroviral drugs. Chemoprophylactic strategies consist of pre-exposure prophyaxis (PrEP) and post-exposure prophyaxis (PEP) [2,3]. PEP involves taking one or more anti-retroviral drugs, preferably within 72 hours of known or suspected exposure to HIV whether through sexual activity, drug use or occupational exposure. In contrast, PrEP is the use of recommended anti-retroviral therapies by selected individuals in the population who are at risk of HIV infection. However, the efficiency in preventing infection in those individuals that take PrEP is correlated with following an appropriate adherence to treatment, a conclusion that has been also observed in studies that have assessed the efficacy of PrEP in "real world" settings [4]. Adherence to treatment and sexual behaviour is also crucial for PEP efficacy [5]. Therefore, attitudes, beliefs and poor awareness towards sex behaviours, risk of STI infection and use of HIV chemoprophylaxis drugs are factors that could impact on the efficacy of these treatments. These factors, including the necessity to engage different communities particularly those in high risk of HIV infection, should be taken into consideration to develop and implement appropriate biomedical and public health interventions [6-8].

Students training to work in subjects allied to medicine or healthcare should have a good working knowledge of routes of HIV infection and anti-HIV strategies. To provide an engaging and productive teaching strategy to train students in these skills and to promote awareness of HIV chemoprophylaxis, we have developed a workshop to teach students how to implement a public health strategy to address HIV infection and the use of PEP/PrEP.

\section{METHODOLOGY}

Students enrolled in the BMedSci undergraduate degree at DMU participated in the workshop delivered as part of the teaching in the level 5 (year 2) module 'Evidence Based Medicine' $(n=41)$. This module was an appropriate platform to deliver the workshop, as the content incorporated and applied fundamental concepts being studied in the module through the use of problem-based learning and reflection to re-inforce the teaching. This cohort of students is also an appropriate testing ground for this type of workshop as many of the students are preparing for a career in subjects allied to medicine and the healthcare sector. An appreciation and ability to design infection prevention strategies are important skills for such students to develop. The students completed a 2 hour research-led workshop designed to identify interventions to reduce HIV infection rates in the Leicester community (including DMU students) following evidence-based public health methodology. To prepare students for the workshop, the students received a one hour lecture with up-to-date information about the HIV virus (including recent articles on HIV accessible as internet links), infection routes and prevention (including HIV chemoprophylaxis), focusing on England and the UK Midlands in particular (the UK region in which DMU is based). The current status regarding PrEP and the UK National Health Service (NHS) was also presented to encourage reflection and discussion amongst the students.

The learning objectives for the workshop were as follows:

- Define evidence-based public health and distinguish it from evidence-based medicine

- Become familiar with key concepts in evidence-based public health

- Apply key concepts to tailoring a public health intervention

Students designed their interventions in small groups and then discussed them with the rest of the class and the academic lead who collectively and constructively critiqued the work. A feedback questionnaire was distributed immediately after completion of the workshop consisting of a short series of 11 Likert (strongly agree; agree; neither agree nor disagree; disagree; strongly disagree) questions (Table 1) and free text questions to solicit comments. The questionnaire was designed based in following successful experiences by our team [9]. Ethical approval was provided by the Research Ethics Committee at DMU (Ref. 1729). 
Table 1. Workshop feedback survey.

Likert questions 1-11 (free text comment boxes were available for each question)

Free text comment questions 12 and 13.

\begin{tabular}{|ll|}
\hline 1 & The content was relevant to the module \\
\hline 2 & The duration of the workshop was appropriate \\
\hline 3 & Overall, I enjoyed the workshop \\
\hline 4 & The workshop was easy to understand \\
\hline 5 & I understand how to perform an evidence based public health study to identify public \\
\hline 6 & My knowledge of public health has improved \\
\hline 7 & The workshop on HIV helped me understand the need for global intervention \\
\hline 8 & I would be able to establish some public health interventions to reduce HIV transmission \\
\hline 9 & I am satisfied with the workshop provided \\
\hline 10 & The knowledge learnt in the workshop will help me in my future career \\
\hline 11 & I would recommend the incorporation of more similar workshops within my programme \\
\hline 12 & What would you add or remove from this training? \\
\hline 13 & Any other comments or suggestions for future years? \\
\hline
\end{tabular}

\section{RESULTS AND DISCUSSION}

The BMedSci students showed substantial interest in this topic, indicated by the extensive discussions generated during the workshop. Overall, the workshop was positively received by the students, revealed by the favourable responses from the class with $92 \%$ of showing satisfaction with the workshop, all students indicating that the workshop was relevant to the module and $85 \%$ of participants recommending inclusion of such workshops in other modules (Fig.1). The workshop survey also revealed that $85 \%$ of participants indicated understanding the necessity of a global intervention to fight HIV and $92 \%$ of students reported to have learnt how to use evidence-based public health to establish interventions to reduce HIV transmission (Fig.1). Uniformly, the participants reported that the workshop had improved their knowledge of public health. Only one question generated notable negative responses which related to the duration of the workshop where $46 \%$ of the respondents did not agree that the workshop was of an appropriate length (Fig.1). A summary of the free text comments coupled with ad hoc verbal feedback from the students to the workshop academic lead indicated that issues with the length of the workshop was perhaps related to the number of articles to be read and discussed in the time available. More discussion and the use of videos and presentations was preferable as opposed to lengthy consideration of articles. Thus, the delivery of information and discussion items through the use of videos was also suggested, an example would be videos of individuals describing personal experiences of PrEP/PEP. We incorporated a range of articles as one of the objectives of the workshop was to provide students with research skills including how to perform an appropriate literature review as well as how to review the evidence to design appropriate biomedical and public health interventions.

Only $39 \%$ of the participants indicated enjoyment of the module, this may be related to the length of the workshop, although precise information was unavailable from the survey. During the group discussions about possible interventions, some degree of confusion was evident with the students with an apparent lack of knowledge regarding transmission routes for HIV and public resources available in the community for earlier diagnosis of infection. The BMedSci students also showed a lack of knowledge of preventative measures (PrEP and PEP) and who should be screened for HIV and when. Since the student cohort to which the workshop was delivered have aspirations to work in professions allied to medicine, practice medicine or pursue other healthcare-related careers, this lack of knowledge regarding routes of infection and infection control is in need of remediation and training, an issue addressed by the workshop described here. 


\section{CONCLUSIONS}

The overall high level of satisfaction and knowledge acquisition/improvement achieved by the HIV training workshop points to the utility of this approach in evidence-based medicine teaching for future healthcare professionals. The current workshop structure can undergo further modification, such a rebalancing the length of the workshop and the types of training materials provided. This and other similar workshops developed by the teaching team could serve as a basis for developing multiple other workshops to embed in modules delivering teaching to trainee health professionals, including those in biomedical and environmental science.

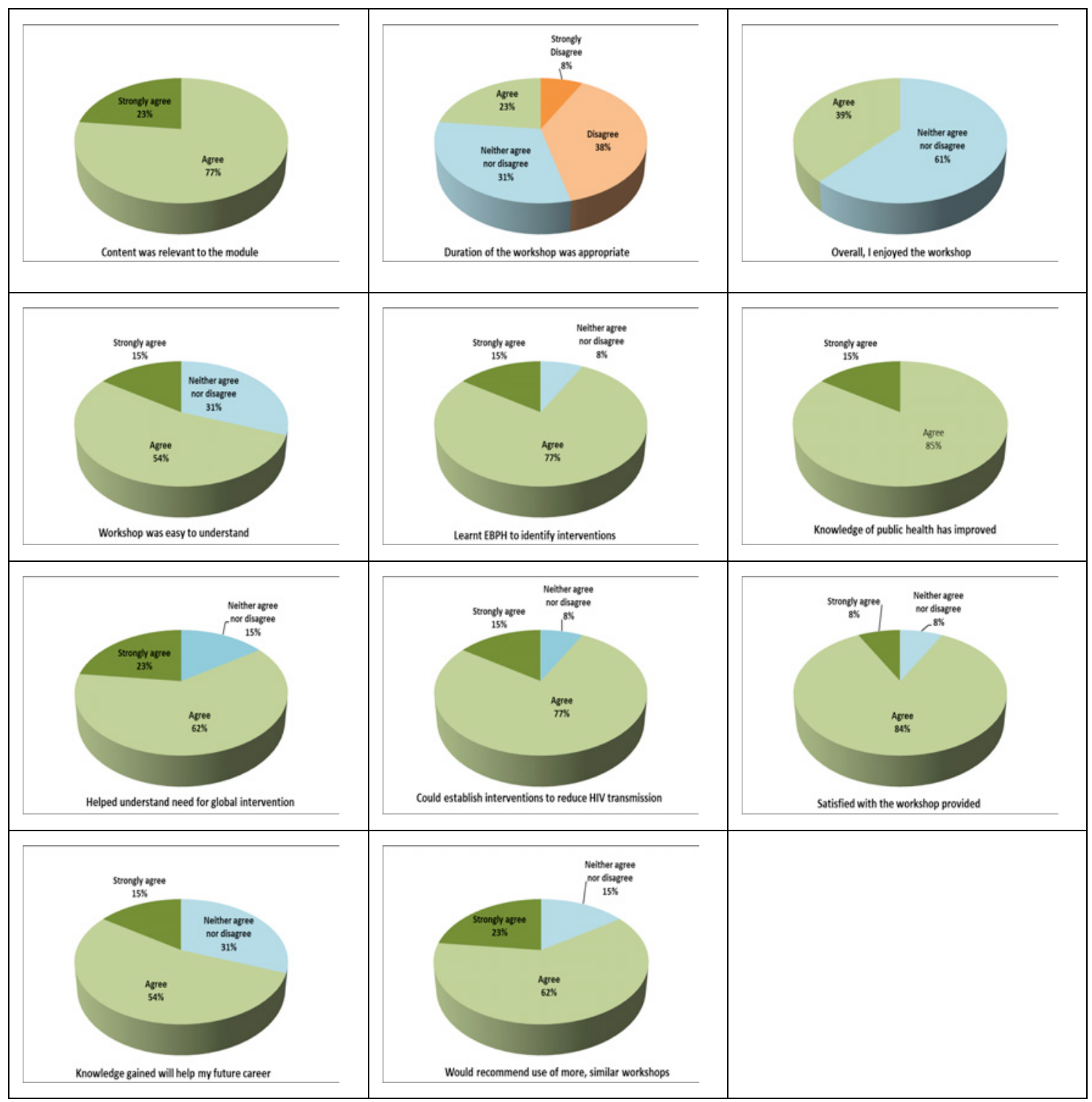

Figure 1: HIV workshop survey results showing percentage of responses for each Likert category. Absence of a Likert category indicates no responses for that category.

\section{REFERENCES}

[1] Public Health England, https://www.gov.uk/government/collections/hiv-surveillance-data-andmanagement.

[2] S. McCormack et al, 'Pre-exposure prophylaxis to prevent the acquisition of HIV-1 infection (PROUD): effectiveness results from the pilot phase of a pragmatic open-label randomised trial', Lancet, vol.387, no.10013, pp.-pp. 53-60, 2016.

[3] B. Sultan et al. 'Current perspectives in HIV post-exposure prophylaxis', HIV AIDS (Auckl). Vol.6, pp.-pp.: 147-158, 2016. 
[4] Marrazzo JM. HIV Prevention: Opportunities and Challenges. Top Antivir Med 2017; 24(4):123126.

[5] Thomas R, Galanakis C, Vézina S, Longpré D, et al. Adherence to Post-Exposure Prophylaxis (PEP) and Incidence of HIV Seroconversion in a Major North American Cohort. PLoS One 2015; 10(11):e0142534.

[6] Eaton LA, Driffin DD, Bauermeister J, Smith H, Conway-Washington C. Minimal Awareness and Stalled Uptake of Pre-Exposure Prophylaxis (PrEP) Among at Risk, HIV-Negative, Black Men Who Have Sex with Men. AIDS Patient Care STDS 2015; 29(8):423-9.

[7] Cahill S, Taylor SW, Elsesser SA, Mena L, Hickson D, Mayer KH. Stigma, medical mistrust, and perceived racism may affect PrEP awareness and uptake in black compared to white gay and bisexual men in Jackson, Mississippi and Boston, Massachusetts. AIDS Care 2017; 29(11):1351-1358.

[8] García M and Harris AL. PrEP awareness and decision-making for Latino MSM in San Antonio, Texas. PLoS One 2017;12(9):e0184014.

[9] Peña-Fernández A., González-Muñoz MJ., Peña MA. Designing training for teaching environmental toxicology to specialized pharmacists. Currents in Pharmacy Teaching and Learning 2015; 7:864-868. 